\title{
Nur gute Schuldner profitieren von niedrigen Zinsen
}

\section{Benedikt Lennartz}

\section{Relevanz}

Damit die Geldpolitik mit niedrigen Zinsen eine Rezession bekämpfen kann, braucht es finanziellen Spielraum bei den Schuldnern. Nur wer beim Kauf einer Immobilie einen hohen Anteil mit Eigenmitteln finanziert, kann die Gunst niedriger Zinsen nützen. Nur gute Schuldner können den Hypothekarkredit weiter aufstocken und Eigenmittel für den Konsum mobilisieren. So können Zinssenkungen die Nachfrage stabilisieren und die Rezession lindern. Wer von vornherein überschuldet ist, hat in der Rezession keinen Spielraum mehr, um mit neuen Krediten den Konsum zu steigern. Systematische Überschuldung nimmt den Zinssenkungen ihre Wirkung und lähmt die Geldpolitik.

\section{Quelle}

Martin Beraja, Andreas Fuster, Erik Hurst und Joseph Vavra (2019), Regional Heterogeneity and the Refinancing Channel of Monetary Policy, Quarterly Journal of Economics, 109-183.

Kann eine klug eingesetzte Geldpolitik genügen, schwere Wirtschaftskrisen zu vermeiden? Die Erfahrung im Jahr 2001 nach dem Platzen der Dotcom-Blase scheint die Wirksamkeit der Geldpolitik zu bestätigen. Die amerikanische Notenbank hatte ihren Leitzins um $5 \%$ abgesenkt und konnte damit die Wirtschaft erfolg-

B. Lennartz $(\bowtie)$

Universität St.Gallen, St.Gallen, Schweiz

E-Mail: benedikt.lennartz@unisg.ch 
reich stützen. In der Finanzkrise ab 2008 senkte sie erneut die Zinsen in ähnlichem Masse. Zusätzliche kaufte sie Anleihen in grossem Umfang auf (Quantitative Easing), um die langfristigen Zinsen zu verringern. Doch diesmal reichte das Eingreifen nicht aus, um eine tiefe Rezession zu vermeiden. Waren also die Zinssenkungen während der Finanzkrise weniger effektiv als sieben Jahre zuvor?

Eine neue Studie der Ökonomen Martin Beraja, Andreas Fuster, Erik Hurst und Joseph Vavra legt diesen Schluss nahe. Die Forscher analysieren einen neuen Wirkungskanal expansiver Geldpolitik. Demnach stützen Zinssenkungen den privaten Konsum auch dadurch, dass Haushalte ihre Immobilienkredite günstiger refinanzieren, was vor allem in den USA weit verbreitet ist. Durch Refinanzierung können verschuldete Haushalte ihre bestehenden Hypotheken mit billigeren Krediten ersetzen und so von einer sinkenden Zinsbelastung profitieren. Wenn die Hauspreise steigen, können sie zudem die Immobilie stärker belasten. Mit steigenden Immobilienwerten können sie höhere Sicherheiten bieten und die Kredite aufstocken. So können sie einen Teil der Eigenmittel, die sie ursprünglich zum Kauf beigesteuert haben, entnehmen und für den Konsum verwenden. Die Wissenschaftler argumentieren, dass dieser Wirkungskanal im Jahr 2001 gut funktionierte, da die Hauspreise überall in den USA gleichmässig stiegen.

Im Jahr 2008 präsentierte sich die Krise jedoch in ganz anderer Art. Die Hauspreise fielen überall und besonders stark in jenen Regionen, die auch den grössten Anstieg der Arbeitslosigkeit zu verkraften hatten. Viele Haushalte waren stark verschuldet und ihr Vermögen, also ihr Haus, hatte stark an Wert verloren. Angesichts schwindender Sicherheiten und hoher Verschuldung konnten die betroffenen Haushalte ihre Kredite nicht mehr weiter aufstocken und hatten keinen Spielraum mehr, um einen Teil der Eigenmittel für Konsumzwecke zu entnehmen. Somit war der zuvor beschriebene Wirkungskanal der Geldpolitik im Jahr 2008 weniger effektiv als sieben Jahre zuvor.

Um diese Argumentation empirisch zu belegen, betrachten die Wissenschaftler zunächst die lokalen Refinanzierungsaktivitäten amerikanischer Haushalte. Die deskriptive Evidenz zeigt, dass kurz nach der Verkündung des ersten geldpolitischen Lockerungsprogramms (QE1) Ende 2008 wesentlich mehr Hypotheken refinanziert wurden. Gleichzeitig sanken die Zinsen langfristiger Kredite. Wo es möglich war, nutzten die Haushalte die günstigeren Kreditbedingungen, um von niedrigeren Zinsen zu profitieren.

Die Forscher zeigen aber, dass es grosse regionale Unterschiede im Verhältnis zwischen dem Kredit zum Wert der Immobilie, also der Beleihungsquote gab. In Philadelphia und Seattle zum Beispiel betrug die Hypothekenschuld eines durchschnittlich verschuldeten Haushalts ca. 70-75\% des Immobilienwerts. In einigen Regionen waren die Immobilienpreise hingegen so stark gesunken, dass sich 
ein grosser Teil der Hauseigentümer in finanziellen Schwierigkeiten befand. So waren z. B. in Las Vegas $70 \%$ der Haushalte stark überschuldet mit Hypothekarschulden, die rund $17 \%$ über dem Immobilienwert lagen.

In Regionen wie Philadelphia und Seattle betrugen die Hypothekarschulden der Haushalte durchschnittlich 70-75\% des Immobilienwertes. In Las Vegas hingegen war bei $70 \%$ der verschuldeten Haushalte die Hypothekarschuld höher als der Wert der Immobilie.

Welchen Einfluss haben diese Verhältnisse auf die Refinanzierungsaktivitäten der Haushalte? Die Forscher teilen hierfür die Regionen nach ihren mittleren Beleihungsquoten in vier Gruppen rein. Dann vergleichen sie die Refinanzierungsaktivitäten der beiden extremen Viertel, das heisst, derjenigen Regionen mit den höchsten und niedrigsten Beleihungsquoten. In beiden Gruppen stieg die Refinanzierungsrate kurz nach der geldpolitischen Lockerung an. In den Regionen mit den geringsten Beleihungsquoten nahm sie besonders deutlich zu. In dem Viertel der Regionen jedoch, in denen die Haushalte im Durchschnitt besonders hoch verschuldet waren, stieg die Refinanzierungsquote deutlich schwächer an. Die Haushalte mussten sich auf eine günstigere Refinanzierung ihrer Hypotheken beschränkten, ohne die Hypothekarkredite aufzustocken und dadurch mehr Geld für Konsum zu mobilisieren.

Nur wer beim Kauf einer Immobilie einen hohen Anteil von Eigenmitteln einsetzt, hat bei sinkenden Immobilienpreisen noch Spielraum, den Hypothekarkredit aufzustocken, um von niedrigen Zinsen zu profitieren. Wenn dagegen die Belehnungsquote schon von vornherein sehr hoch ist, gibt es angesichts der Überschuldung keinen Spielraum mehr. Die Autoren können genau zeigen, in welchem Umfang Haushalte die niedrigen Zinsen nutzen konnten, um mehr Geld für Konsumausgaben zu mobilisieren. Wie zuvor lässt sich beobachten, dass die Haushalte in den Regionen mit der niedrigsten Verschuldung deutlich mehr Eigenmittel durch Refinanzierung herausnehmen konnten als die Haushalte in den Regionen mit der höchsten Verschuldung. Ende 2009 hatten die Haushalte in den Regionen mit geringen Beleihungsquoten rund $8 \mathrm{Mrd}$. \$ mehr an Eigenmittel aus der Refinanzierung ihrer Hypothekarschulden bezogen als die Haushalte in stark verschuldeten Regionen.

In gering verschuldeten Regionen hatten Haushalte um 8 Mrd. Dollar mehr Eigenmittel bezogen als in hoch verschuldeten Regionen.

Um zu zeigen, inwieweit die Refinanzierung und Aufstockung von Hypothekarschulden bei niedrigen Zinsen die Konsumausgaben steigerten, vergleichen 
die Wissenschaftler die Verkaufszahlen von Neuwagen zwischen den stark und schwach verschuldeten Regionen. Autokäufe schwanken üblicherweise stärker als allgemeine Konsumausgaben und nehmen daher bei einem Anstieg der verfügbaren Einkommen besonders stark zu. Tatsächlich stiegen die Autokäufe besonders in den weniger verschuldeten Regionen. Die Ergebnisse deuten also daraufhin, dass die Lockerung der Geldpolitik den Konsum gerade in jenen Regionen anregte, wo die Arbeitslosigkeit niedriger war und die Immobilienpreise vergleichsweise weniger stark gefallen waren. In den Gegenden, welche besonders stark von steigender Arbeitslosigkeit und fallenden Immobilienpreisen betroffen waren und die Überschuldung zunahm, nahmen die Autokäufe hingegen deutlich schwächer zu.

Die Wissenschaftler können nun den Vergleich zur Dotcom-Krise zu Beginn der 2000-er Jahre ziehen. Zunächst dokumentieren sie die empirischen Fakten zum makroökonomischen Umfeld. Während der Rezession 2001 stiegen die Hauspreise im Durchschnitt, wogegen sie 2008 fielen. Zudem waren die regionalen Unterschiede 2008 deutlich höher. Der Zusammenhang von Immobilienpreisen und Arbeitslosigkeit in den verschiedenen Regionen war 2008 sehr viel stärker ausgeprägt als 2001. Mit diesen Erkenntnissen deckt sich, dass die Refinanzierungsquoten im Jahr 2001 ein anderes regionales Muster zeigten als sieben Jahre später. Damals waren es Haushalte in den Regionen mit der höchsten Arbeitslosigkeit, die am häufigsten refinanzierten. Sie brauchten am dringendsten mehr verfügbares Einkommen und konnten von den niedrigen Zinsen profitieren, weil sie dank steigender Immobilienpreisen mehr Sicherheiten bieten und ihre Hypothekarkredite aufstocken konnten. Somit wirkte $2001 \mathrm{der}$ geldpolitische Impuls gerade dort, wo er besonders notwendig war. Nach Abb. 1 waren es die Regionen mit der höchsten Arbeitslosigkeit, in denen die Haushalte deutlich öfter refinanzierten, während es in der Krise 2007-2009 mit fallenden Immobilienpreisen gerade umgekehrt war.

Um die gesamtwirtschaftlichen Effekte expansiver Geldpolitik während beider Rezessionen zu vergleichen, entwickeln die Forscher ein quantitatives Modell und passen es an die makroökonomischen Gegebenheiten vor der Finanzkrise an. Dann simulieren sie, wie sich Zinssenkungen auf Kreditrefinanzierungen und Konsum auswirken. Im Einklang mit der empirischen Evidenz steigen die Refinanzierungsraten und der gesamtwirtschaftliche Konsum besonders in den Regionen mit niedriger Verschuldung. Weil dort die Arbeitslosigkeit geringer und die Haushalte wohlhabender sind, akzentuiert der geldpolitische Impuls tendenziell auch die Ungleichheit des Konsums. Nun passen die Forscher das Modell an die ökonomischen Gegebenheiten des Jahres 2001 an und simulieren dieselbe Zinssenkung wie zuvor. Der Konsumanstieg ist nun knapp doppelt so 


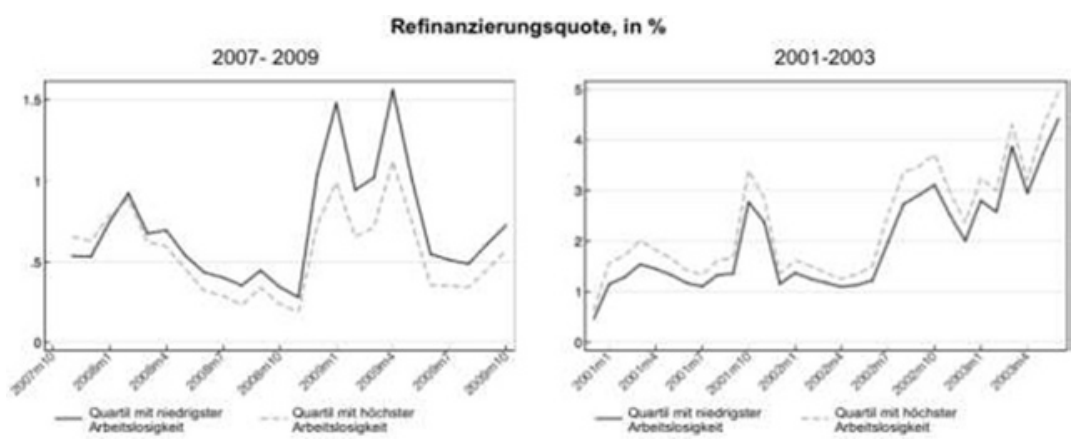

Abb. 1 Refinanzierungsquoten und regionale Arbeitslosigkeit während der Finanzkrise und der Dotcom-Krise

gross wie zuvor. Dies bedeutet, dass die Geldpolitik während der Finanzkrise einen viel stärkeren Effekt gehabt hätte, wenn die Verschuldung der Haushalte ähnlich niedrig gewesen wäre wie 2001. Die Autoren führen im Wesentlichen zwei Gründe an. Zunächst waren 2008 deutlich mehr Haushalte überschuldet. Somit war es ihnen nicht möglich, ihre Schulden weiter zu erhöhen, um von den niedrigen Zinsen zu profitieren. Zusätzlich hatten 2001 diejenigen Haushalte, die nicht überschuldet waren, deutlich niedrigere Kredite. Sie hatten daher mehr Spielraum, bei der Refinanzierung die Hypothekarkredite aufzustocken und mehr Eigenmittel für den Konsum verfügbar zu machen.

Es stellt sich die Frage, welche wirtschaftspolitischen Massnahmen die Geldpolitik wieder effektiver machen könnten. Die Wissenschaftler betrachten in ihrem Modell einen Schuldenschnitt für überschuldete Haushalte. Eine ähnliche Massnahme hatten USA mit dem „Home Affordable Modification Program“ 2009 umgesetzt. Im Modell erhöht ein partieller Schuldenschnitt den Konsum. Es zeigt sich also, dass Zinssenkungen alleine noch nicht ausreichen, um bei starker Überschuldung eine Rezession zu verhindern. Es braucht zusätzliche, gezielte Massnahmen, um die Wirksamkeit der Geldpolitik zu unterstützen. 
Open Access Dieses Kapitel wird unter der Creative Commons Namensnennung 4.0 International Lizenz (http://creativecommons.org/licenses/by/4.0/deed.de) veröffentlicht, welche die Nutzung, Vervielfältigung, Bearbeitung, Verbreitung und Wiedergabe in jeglichem Medium und Format erlaubt, sofern Sie den/die ursprünglichen Autor(en) und die Quelle ordnungsgemäß nennen, einen Link zur Creative Commons Lizenz beifügen und angeben, ob Änderungen vorgenommen wurden.

Die in diesem Kapitel enthaltenen Bilder und sonstiges Drittmaterial unterliegen ebenfalls der genannten Creative Commons Lizenz, sofern sich aus der Abbildungslegende nichts anderes ergibt. Sofern das betreffende Material nicht unter der genannten Creative Commons Lizenz steht und die betreffende Handlung nicht nach gesetzlichen Vorschriften erlaubt ist, ist für die oben aufgeführten Weiterverwendungen des Materials die Einwilligung des jeweiligen Rechteinhabers einzuholen.

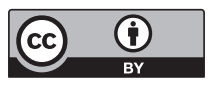

